\title{
Lidil
}

Revue de linguistique et de didactique des langues

$63 \mid 2021$

Littératie numérique et didactique des langues et des cultures

\section{Former à la littératie numérique de futurs enseignants de FLE : une approche par genres numériques}

Training Future FFL Teachers in Digital Literacy: A Digital Genre-Based Approach

\section{Christelle Combe}

\section{CpenEdition}

\section{Journals}

Édition électronique

URL : http://journals.openedition.org/lidil/8959

DOI : $10.4000 /$ lidil.8959

ISSN : 1960-6052

\section{Éditeur}

UGA Éditions/Université Grenoble Alpes

\section{Édition imprimée}

ISBN : 978-2-37747-283-3

ISSN : $1146-6480$

\section{Référence électronique}

Christelle Combe, «Former à la littératie numérique de futurs enseignants de FLE : une approche par genres numériques », Lidil [En ligne], 63 | 2021, mis en ligne le 30 avril 2021, consulté le 30 avril 2021. URL : http://journals.openedition.org/lidil/8959; DOI : https://doi.org/10.4000/lidil.8959

Ce document a été généré automatiquement le 30 avril 2021

(C) Lidil 


\title{
Former à la littératie numérique de futurs enseignants de FLE : une approche par genres numériques
}

\author{
Training Future FFL Teachers in Digital Literacy: A Digital Genre-Based \\ Approach
}

Christelle Combe

\section{Introduction}

1 Avec la révolution numérique, enseigner les langues signifie aussi, désormais, prendre activement en considération l'écosystème numérique dans lequel les individus interagissent. En outre, des compétences numériques, en constante évolution, sont indispensables à acquérir et à actualiser pour les futurs enseignants de français langue étrangère (FLE). Depuis plusieurs années, des modules qui visent à rendre les étudiants capables d'intégrer le numérique de manière critique dans leur enseignement futur (Mangenot, 2017) sont mis en place dans les formations. Dans cette perspective, nous proposons dans notre formation de futurs professionnels du FLE deux cours complémentaires qui se veulent une réflexion « sur » et une pratique « du » numérique avec une approche particulière par genres numériques.

2 Notre ancrage théorique est à la fois linguistique et didactique. Il se fonde sur les notions de littératies médiatique, multimodale et numérique et s'inscrit dans la continuité des liens qui unissent l'analyse du discours (AD) et la notion de genre discursif à la didactique du FLE que nous revisitons à partir de l'analyse du discours numérique $(\mathrm{ADN})$ et de la notion de genre numérique appliquée à la didactique des langues (DDL) ${ }^{1}$. Après avoir exposé notre démarche méthodologique, nos analyses mettront en évidence comment, au sein de ces deux cours, les étudiants de master 
s'emparent des différents éléments de la formation à la littératie numérique à travers notamment l'analyse du discours numérique et l'appropriation de trois genres numériques émergents de la fonction enseignante : le selfie vidéo tutoral (Combe \& Cappellini, 2021), la consigne en ligne (Combe Celik, 2010 ; Cappellini \& Combe, 2017) et enfin le e-portfolio de développement professionnel (Karsenti \& Collin, 2012; Combe, 2020).

\section{Ancrage théorique}

3 Après avoir défini les littératies médiatique, multimodale et numérique, nous présenterons le nouveau cadre de l'analyse du discours numérique (Paveau, 2017) ainsi que la notion de genre numérique que nous appliquons en DDL.

\subsection{Littératies médiatique, multimodale et numérique}

Depuis la fin du $\mathrm{xx}^{\mathrm{e}}$ siècle, la notion de littératie s'est largement complexifiée. Pour rendre compte de la pluralité et de la diversité des modes (visuel, oral, écrit, etc.), des langues (plurilinguisme), des pratiques, des situations, des contextes et des technologies, le New London Group crée le terme de «multilittératie »(1996). Pour caractériser les nouvelles compétences dont l'individu doit disposer pour «évoluer de façon critique, créative, autonome et socialisée dans l'environnement médiatique contemporain" (Lebrun et coll., 2012, p. 47), Lebrun et coll. parlent d'abord de « littératie médiatique». Puis, en 2018, Lacelle et coll. définissent "la littératie médiatique multimodale en contexte numérique » comme :

[...] la capacité d'une personne à mobiliser adéquatement, en contexte communicationnel synchrone ou asynchrone, les ressources et les compétences sémiotiques modales et multimodales les plus appropriées à la situation et au support de communication, à l'occasion de la réception et (ou) de la production de tout type de message. (p. 8)

5 La multimodalité est alors admise, d'une part, comme le recours à divers modes d'expression (oralo-verbal, posturo-mimo-gestuel, iconique, audio, vidéo, tactile, etc.) pour créer du sens, mais aussi et surtout lorsque ces modes sont employés de manière conjointe pour former des touts multimodaux signifiants (Jewitt et coll., 2016).

Quant à Ollivier (2018), il définit la littératie numérique comme le fruit de l'interconnexion de trois grandes littératies comprises dans un cadre critique et éthique :

- la littératie technologique qui est la capacité à identifier des outils et ressources numériques et à savoir les utiliser ;

- la littératie de la construction de sens qui regroupe la littératie des médias, la littératie informationnelle et la littératie du visuel ;

- la littératie de l'interaction qui regroupe la littératie de la communication médiatisée par les technologies, la littératie de la collaboration et la littératie de la participation.

7 Le cadre critico-éthique prend en compte les notions de confidentialité, de sécurité, deconstruction et gestion de l'identité numérique, de santé humaine et environnementale, et favorise les pratiques numériques inclusives. Il rejoint en ce sens la notion de citoyenneté numérique (Frau-Meigs et coll., 2017). Sorte d'hyperonyme, 
littératie numérique regroupe donc l'ensemble des littératies propres à un écosystème numérique.

8 À l'échelle de l'Europe, face aux défis qu'implique l'ère numérique, un cadre de référence européen pour les compétences numériques des enseignants (DigCompEdu²) a été rédigé.

Confrontés à des demandes en évolution rapide, les enseignants doivent maitriser des compétences de plus en plus étendues et sophistiquées. L'omniprésence des dispositifs numériques et le devoir qui leur incombe d'aider les élèves à acquérir des compétences numériques exigent des enseignants qu'ils développent leur propre compétence numérique. (2017, p. 4)

Toutefois aux compétences technosémiopédagogiques des enseignants de langue (Guichon, 2012), des connaissances technosémiodiscursives sont un préalable indispensable. L'ADN et une approche par genre nous semblent un angle d'attaque prometteur.

\subsection{De l'analyse du discours à l'analyse du discours numérique en didactique des langues}

Depuis de longues années, la didactique du FLE convoque l'AD pour en faire un instrument au service de l'analyse des supports de formation et des discours tenus par les acteurs de formations en langue (Claudel, 2017).

11 Aujourd'hui, nombreux sont les supports de formation extraits d'Internet et du web social et de plus en plus fréquentes sont les interactions par écran. Dans les formations de master FLE des modules forment à exploiter Internet et le web social dans une approche par tâches et s'organisent autour de télécollaborations selon des modalités différentes - asynchrones sur forums ou synchrones par visioconférence (Develotte \& Mangenot, 2010). Toutefois, les seuls outils de l'AD ne semblent plus suffisamment adaptés aux technodiscours (productions discursives natives) qui présentent des traits spécifiques :

[...] en ligne, ils sont composites (co-constitués de langagiers et de technologique), délinéarisables (par les liens hypertexte), relationnels (tout énoncé en ligne est lié à un autre énoncé, un appareil et un internaute), investigables (conséquence de la relationalité et de la structure algorithmique du web), imprévisibles (conséquence de la composition et de la relationalité) et augmentables (du fait de la nature participative du web et d'outils dédiés à l'écriture collective). (Paveau, 2017, p. 335)

Et il nous semble pertinent de recourir au cadre plus adapté de l'ADN ainsi définie : L'analyse du discours numérique consiste en la description et l'analyse du fonctionnement des productions langagières natives d'internet, et plus particulièrement $\mathrm{du}$ web 2.0, dans leurs environnements de production, en mobilisant à considération égale les ressources langagières et non langagières des énoncés élaborés. (Ibid., p. 27)

12 Ainsi, les futurs enseignants de langue auront les outils nécessaires pour analyser dans une perspective critique le discours numérique, un objet familier en apparence et pourtant complexe, multimodal et plurisémiotique, afin d'en faire un objet didactique. Il apparait en effet indispensable qu'ils soient formés aux nouvelles formes de lecture, d'écriture et de publication propres à un environnement numérique. 


\subsection{De la notion centrale de genre à celle de genre numérique}

13 L'AD accorde un rôle clé aux genres de discours qui sont considérés comme des dispositifs de communication, de nature à la fois sociale et linguistique (Maingueneau, 2012). Et comme le souligne Claudel (2017) :

[...] une démarche d'AD en didactique vise non seulement la compréhension de genres discursifs, mais également la production de ces mêmes genres à travers le réemploi des principales opérations (énonciatives, discursives, cognitives, etc.) constitutives des matrices observées. (\$34)

14 L'ADN propose de saisir les genres de discours numériques tels qu'ils se développent dans leur environnement en tenant compte de leur dimension composite, issue d'une coconstitution du langagier et du technologique (Paveau, 2017). Aussi, il nous semble important de former les futurs enseignants de langue non seulement à l'analyse des différents genres numériques (Combe, 2019) dans lesquels ils pourront puiser pour étayer leurs cours de langue (supports, etc.), mais aussi de les initier plus particulièrement aux genres numériques propres aux discours enseignants dont le selfie vidéo, la consigne en ligne et le e-portfolio de développement professionnel.

Une première analyse de selfies vidéos tutoraux montre que l'on retrouve

[...] les caractéristiques de la scène générique du selfie mais aussi du vlogue éducatif ou humoristique avec toutefois une approche originale de chaque tuteur qui met ses compétences de vidéaste amateur à contribution afin de construire un ethos singulier (Combe \& Cappellini, 2021, p. 44).

Dans un cadre d'enseignement en ligne, Combe Celik (2010) parle de la consigne en ligne comme d'un genre en pleine évolution et précise que la maitrise de ce genre par celui qui la rédige est une des conditions à la bonne réussite de la tâche et au suivi dans son ensemble.

Pour être efficace dans une formation en ligne, la consigne doit se situer à la frontière entre la consigne traditionnelle et le message de courrier électronique. En effet, elle doit à la fois conserver les caractéristiques d'une consigne, à savoir se centrer sur le référent et être rigoureusement structurée. [...] Mais elle doit aussi être un premier dialogue entre le tuteur et les étudiants, une première opportunité pour lui d'établir une relation de proximité avec ses apprenants, de poser les premiers jalons d'une dynamique de groupe qu'il n'aura de cesse de relancer tout au long de l'année. Le tuteur peut à bon escient utiliser les autres modes (audio, graphique, etc.) afin non seulement de rompre la monotonie de l'échange mais également de montrer par l'exemple aux étudiants le potentiel de la plateforme. (p. 235)

17 Enfin, le genre du e-portfolio :

[...] est un genre hybride qui se développe sur une plateforme numérique et conjugue les fonctions d'apprentissage réflexif en continu, de mise en réseau et de mise en valeur des travaux réalisés et compétences acquises. Il se caractérise par une écriture et un design numérique multimodal (possibilité de mêler du texte, de l'audio, de la vidéo, de l'iconographie, des liens hypertextuels et d'organiser les rubriques et le rendu à l'écran des différents espaces discursifs). Il présente un caractère extime ${ }^{3}$ et continu. Il permet la mise en réseau par affinité, par regroupement mais aussi vers le monde professionnel extérieur. Il donne la possibilité à l'étudiant d'exprimer sa créativité et de s'engager dans un processus de réflexion sur son apprentissage à travers la production d'une œuvre numérique esthétique, évolutive et personnelle. (Combe, 2020, p. 112). 
18 Ainsi, c'est à partir des notions de littératies médiatique, multimodale et numérique et du cadre conceptuel de l'ADN et de la notion centrale de genre numérique que la formation des futurs professionnels de FLE, présentée ci-après, a été pensée.

\section{Démarche méthodologique}

19 Notre démarche méthodologique est qualitative et descriptive. Cette étude est une recherche-action exploratoire qui s'élabore, s'enrichit, se modifie au fur à mesure de l'expérience pédagogique et des différentes analyses conduites. Enseignante au sein du master et chercheuse, nous améliorons notre pratique et l'offre de formation à partir des différents cycles d'expérimentation qui sont menés sur les cohortes qui se succèdent. En tant que recherche-action, elle est nécessairement ouverte puisqu'il est impossible de déterminer à l'avance le cours de l'action, le nombre de cycles impliqués, ni même le résultat final (Lacelle et coll., 2018).

\subsection{Présentation des données et du corpus d'étude}

Les données ont été recueillies auprès de deux cohortes de 20 étudiants chacune, au cours de deux années universitaires (de 2018 à 2020). Sept étudiants (Alain, Carine, Frédéric, Léa, Marie, Natacha et Tabatha) pour la première année et cinq (Agnès, Carine, Céline, Emma et Emmanuelle) pour la deuxième année ont donné leur accord pour l'exploitation de leurs données. Le corpus d'étude se compose donc des productions multimodales en contexte numérique que ces douze étudiants ont été amenés à concevoir tout au long de leur master, à savoir : une vidéo de présentation de soi, des consignes en ligne sur une plateforme d'enseignement et enfin des e-portfolios de développement professionnel. Ces données premières sont enrichies des synthèses réflexives qu'ils ont rendues à la fin des deux cours. Notre étude étant à visée descriptive, nous illustrerons les analyses à partir d'exemples signifiants et encore non étudiés. Tous les extraits seront cités dans leur orthographe d'origine.

\subsection{Modalités d'analyse}

Dans un premier temps, nous avons observé la mise en discours numérique (Cappellini \& Combe, 2017) des productions réalisées par les étudiants à partir des outils de l'ADN et des catégories de mise en écran, mise en rubriques, mise en média et mise en discours (Develotte, 2006). Nous avons cherché à voir comment les étudiants réinvestissent dans leurs productions tutorales les caractéristiques des genres émergents qu'ils ont pu étudier en amont à travers l'analyse de documents numériqués ${ }^{4}$. Nous pourrons voir par exemple s'ils maitrisent les matrices discursives dans lesquelles ils inscrivent leur production, mais aussi comment ils parviennent à créer et à faire évoluer ces genres émergents. Une analyse de contenu (Bardin, 2013) pour les synthèses réflexives permet d'étayer nos observations. 


\section{Projet pédagogique}

22 Dans un premier temps, nous présentons du point de vue de leur conception didactique ces deux cours complémentaires l'un de l'autre: «Développer ses compétences en littératie numérique et les intégrer à sa pratique professionnelle » et «Concevoir et tutorer un cours de langue en ligne ».

\subsection{Développer ses compétences en littératie numérique et les intégrer à sa pratique professionnelle}

L'objectif de ce premier cours, qui a lieu au semestre 1, est de faire saisir aux étudiants la complexité et l'importance des notions de littératies médiatique, multimodale et numérique en tant que futurs professionnels du FLE tout en les amenant à accroitre leur propre littératie numérique afin qu'ils soient à même de développer celle de leurs futurs apprenants au cours de leur enseignement de la langue.

Les étudiants sont amenés à acquérir les compétences suivantes :

- analyser un document numériqué à l'aide des outils de l'ADN ;

- découvrir et produire deux genres numériques de présentation de soi : le selfie vidéo tutoral et le e-portfolio de développement professionnel ;

- construire un scénario pédagogique ${ }^{5}$ intégrant la réception et production d'un genre numérique identifié et des compétences transversales en littératie numérique.

Après une réflexion sur les changements induits par le numérique, sur le modèle d'une biographie langagière, les étudiants rédigent leur biographie numérique. Ils sont ensuite initiés à l'ADN. À partir de la notion centrale de genre numérique, ils doivent alors produire l'analyse multimodale d'un document numériqué de leur choix. Puis, à partir de l'approche socio-interactionnelle (ollivier, 2018), ils élaborent un scénario pédagogique qui travaille un genre numérique et intègre des compétences apprenantes en littératie numérique. Parallèlement, à partir des notions de présentation de soi en ligne et d'ethos numérique, ils sont invités à réaliser un selfie vidéo tutoral pour initier le projet pédagogique du cours " Concevoir et tutorer un cours de langue en ligne ». Est ensuite abordée la notion de e-portfolio de développement professionnel du point de vue de la littératie numérique ; les étudiants débutent alors l'élaboration numérique de leur propre e-portfolio ${ }^{6}$.

\subsection{Concevoir et tutorer un cours de langue en ligne}

26 Le second cours, qui a lieu au semestre 2, relève d'une pédagogie de projet et est organisé autour d'une télécollaboration du type "Le français en (première) ligne " (Develotte \& Mangenot, 2010). Dans ce projet, les étudiants de master conçoivent et tutorent des activités en ligne pour des apprenants de FLE de l'Université de Californie Berkeley de niveau B1-B2.

L'objectif de ce cours est d'initier les étudiants à l'enseignement du FLE en ligne au sein de différents environnements (principalement un forum de discussions sur une plateforme d'enseignement en ligne de l'université partenaire et une plateforme multimodale de visioconférence). Il est conçu comme un prolongement du cours précédent et une mise en application des différents acquis en tenant compte fortement 
toutefois du partenaire et de ses contraintes et exigences, autrement dit du contexte d'enseignement. Les étudiants sont conduits à acquérir les compétences suivantes :

- sélectionner et didactiser des documents numériqués pour des cours en ligne ;

- formuler des consignes et des feed-back en asynchrone et synchrone ;

- prendre conscience de son ethos en ligne et l'adapter à la situation de communication et aux différents environnements numériques.

La rencontre entre les tuteurs et leurs apprenants s'élabore progressivement tandis que l'ethos professionnel et numérique des tuteurs se met aussi peu à peu en place. Ils profitent également de cette expérience pour alimenter leur e-portfolio, notamment cette fois dans sa partie réflexive.

\section{Analyses des productions}

Dans un second temps, nous observons comment les étudiants se sont approprié l'analyse d'un document natif du numérique et les trois genres tutoraux qu'ils ont été amenés à produire.

\subsection{De l'analyse d'un document numériqué à la mise en discours numérique de son e-portfolio}

Les étudiants ont librement choisi leur document numériqué à analyser et donc le genre afférent. La première année, nous avions davantage orienté leur réflexion autour de genres numériques majeurs comme le mème (Alain et Léa), le vlogue (Natacha et Frédéric), le e-portfolio (Marie et Tabatha). L'année suivante, nous avons laissé les étudiants opter pour le document numériqué de leur choix, les incitant même à l'originalité, afin de pouvoir le décrire dans toute sa complexité et d'en saisir les nuances numéricocontextuelles au-delà des caractéristiques technosémiodiscursives. C'était ainsi l'occasion de permettre aux étudiants de partager leurs explorations numériques et pour le groupe de découvrir des applications et réseaux inconnus ${ }^{7}$. Les étudiants ont alors retenu le site de regroupement communautaire Reddit, le réseau social Pinterest, le site d'hébergement communautaire Airbnb, le e-portfolio LinkedIn et l'application de réseautage Amino.

31 Dans une première étape, les étudiants ont décrit à partir du lexique de l'ADN ce document numériqué dans sa complexité et sa multimodalité, mettant en œuvre leurs compétences technosémiodiscursives comme le schéma ci-dessous, extrait de la synthèse réflexive de Céline, l'illustre : 
Figure 1. - Schéma de Céline analysant le genre du e-portfolio de développement professionnel Linkedln.

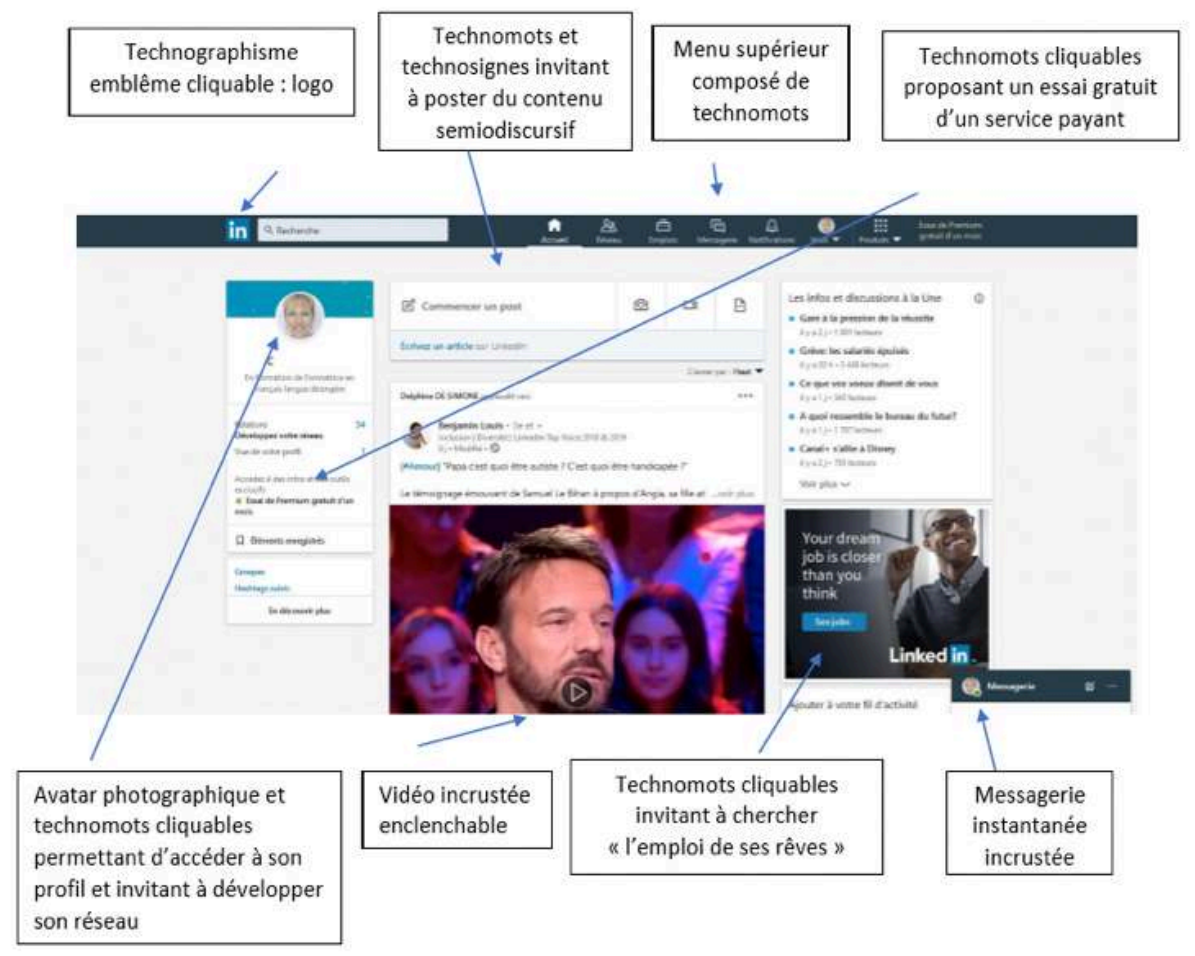

L'analyse détaillée de Céline met en évidence l'organisation technosémiodiscursive d'un profil LinkedIn, mais ne s'arrête pas à ce seul aspect descriptif. L'étudiante porte un regard critique sur le fonctionnement de ce genre d'e-portfolio proposé par cette plateforme en particulier. Son schéma souligne des éléments clés comme l'invitation à poster du contenu, à développer son réseau, à chercher «l'emploi de ses rêves ». Et elle montre dans sa synthèse réflexive sa capacité à décrypter le fonctionnement de ce réseau social :

Le site est gratuit mais son modèle économique repose sur 3 sources de revenus : la publicité, les abonnements et les services payants de recrutement et de formations en ligne. Ces services sont d'ailleurs déclinés sous le terme de « produits » [...] : le terme est révélateur8. Aussi, sous ses abords de réseau social, fonctionnant a priori sur la mise en relation, la collaboration, le partage d'informations, se cache en réalité une machine commerciale bien huilée. [...] Ce service propose, entre autres de voir qui a consulté notre profil (en l'occurrence des recruteurs potentiels). Il repose sur plusieurs ressorts psychologiques : la curiosité, l'égo («telle grosse entreprise a regardé mon profil»), ou la fébrilité/insécurité lorsqu'on est en recherche d'emploi. Les technomots cliquables apparaissent à deux endroits sur la même page: en français et en anglais (« essai gratuit»- «Try Premium for free »), ce qui sonne comme une injonction à souscrire au service. (Extrait de la synthèse réflexive de Céline)

33 Cette compétence d'analyse technosémiodiscursive s'avère utile aux étudiants dans la mise en discours numérique de leur propre e-portfolio.

Le e-portfolio de développement professionnel est un genre numérique essentiel à l'étudiant, futur professionnel, dans une perspective de formation tout au long de la vie. Abordé sur le plan de la littératie numérique au premier semestre, il se poursuit sur le plan du travail réflexif tout au long du master. 


\section{propre création :}

Apprendre à analyser des documents numériqués nous permet de mieux comprendre les choix effectués par leurs concepteurs. Cela nous a donc aidé lorsque nous devions concevoir notre propre site e-portfolio. (Extrait de la synthèse réflexive d'Emmanuelle)

Le plan de la synthèse réflexive d'Agnès matérialise comment l'appropriation des différents concepts de l'ADN (comme la mise en discours numérique, la question de l'extimité et de l'ethos) a servi la réalisation de son e-portfolio :

Tableau 1. - Plan de la synthèse réflexive d'Agnès sur l'élaboration de son e-portfolio.

\begin{tabular}{|l|l|}
\hline 1. Réflexions avant de matérialiser le & $\begin{array}{l}\text { 1.1. Les toutes premières réflexions } \\
\text { e-portfolio }\end{array}$ \\
& $\begin{array}{l}\text { 1.2. La carte mentale } \\
\text { 1.4. Le choix du « site de propulsion » } \\
\text { 1.5. Le choix de la charte graphique et de « sa palette } \\
\text { de couleurs » }\end{array}$ \\
\hline $\begin{array}{l}\text { 2. D'un e-portfolio d'apprentissage vers un } \\
\text { e-portfolio de présentation }\end{array}$ & $\begin{array}{l}\text { 2.1. Mise en écran | mise en media | mise en rubrique } \\
\text { mise en discours } \\
\text { 2.2. Réflexions autour de l'usage } \\
\text { 2.3. Extime et ethos }\end{array}$ \\
\hline
\end{tabular}

Dans l'extrait suivant, à partir de ses acquis théoriques, Emmanuelle montre également comment elle a pensé et défini sa « scénographie numérique »:

Le choix des couleurs et des images fait donc partie de ma "scénographie" numérique. Il y a donc une page d'accueil dans laquelle se trouvent deux rubriques qui mènent à d'autres pages : « mon parcours » et « mes passions » : on peut cliquer sur le technomot « en savoir plus » pour accéder aux pages correspondantes (pages où il $\mathrm{y}$ a le plus à dire, nécessitant plus d'explications). Ces deux parties contiennent chacune trois sous-parties afin de les expliciter. Il est aussi possible d'accéder aux pages en cliquant sur les technomots en haut de mon site sur le bandeau de présentation pour éviter de dérouler tout le site. (Extrait de la synthèse réflexive d'Emmanuelle)

Tandis que Céline, qui a analysé le genre du e-portfolio dans sa version proposée par LinkedIn, fait le lien avec son propre e-portfolio autour de la question prégnante de l'identité numérique et de ce que l'on donne à voir :

Moi qui suis d'ordinaire plutôt discrète, je devais me dévoiler. J'avais l'impression d'étaler ma vie, de m'exhiber en quelque sorte. Mais voilà, le but du e-portfolio est justement de se présenter'. Cela induit une certaine responsabilité : que puis-je montrer pour donner envie de lire, me contacter, de collaborer avec moi ou m'employer? D'autant que je ne suis pas graphiste ni webmaster. Je devenais donc pro-am à mon tour. D'autre part, jusqu'où pouvais-je aller pour cela, tout en protégeant un tant soi peu mon intimité ? À l'heure où l'on parle beaucoup de protection des données personnelles, je devais les livrer sciemment. Cela sonnait en moi comme un dilemme. Moi qui ne produis presque rien sur les réseaux du type Facebook, à part quelques « likes » ou un partage d'image amusante. Pas de photo, pas de coordonnées, pas de discours 
personnel, je devais me jeter à l'eau. L'avantage est que j'avais la liberté de choisir ce que je voulais montrer... ou pas. (Extrait de la synthèse réflexive de Céline)

Cet extrait met bien en évidence l'injonction paradoxale de cette formation au numérique ainsi que la difficulté de l'exercice pour les étudiants qui sont parfois contraints d'aller au-delà de leur nature propre tout en montrant leur maitrise du cadre critico-éthique de la littératie numérique. Ce cadre apparait alors toujours plus indispensable à la formation et semble assez bien acquis par Céline, comme en témoignent les interrogations auxquelles elle-même se confronte.

Ainsi, les étudiants ont transféré leurs compétences d'analyse dans la mise en pratique du genre du e-portfolio de développement professionnel. Ils ont aussi su réinvestir leurs acquis théoriques dans le genre tutoral de la consigne en ligne sur un forum de discussions.

\subsection{Vers la maitrise d'un premier genre tutoral : la consigne en ligne}

41 Souvent, l'espace de production discursive d'une consigne en ligne sur un forum de discussions est similaire à l'écriture d'un mail sur une messagerie en ligne comme Gmail ou d'une publication sur une plateforme comme Moodle par exemple. Le format et les outils de mise en discours numérique sont donc familiers aux étudiants tant dans leurs pratiques sociales qu'étudiantes ${ }^{10}$. Toutefois, la mise en discours numérique d'une consigne en ligne sur un forum de discussions exige la maitrise d'un certain nombre de critères récurrents.

En premier lieu, elle s'inscrit généralement dans un fil de discussion ouvert par l'étudiant-tuteur. Entre la première et la deuxième cohorte, on observe la généralisation d'un seul fil de discussion par étudiant et apprenant, intitulé le plus souvent de la manière suivante : «Tutorat avec [Prénom de l'étudiant-tuteur] ». Certes, ce titre manque un peu d'originalité et ne répond pas à la fonction d'accroche (Combe Celik, 2010) qui devrait être la sienne, mais il donne la possibilité à l'apprenant de retrouver directement, grâce à une recherche par mots-clés, le nom de son tuteur dans la liste des messages, la télécollaboration étant organisée en binômes. On note aussi que la plupart des étudiants ont personnalisé leur avatar par une photographie ou encore un émoticône à leur effigie.

Le message commence par une formule d'ouverture familière et amicale ("salut", «bonjour ", " coucou » parfois suivi du prénom de l'apprenant), et se termine par une formule de clôture qui engage à poursuivre l'interaction ("à très vite», "à très bientôt ", "à tout à l'heure " généralement ponctuée d'un point d'exclamation). Ces moments ménagent l'aspect socio-affectif de la relation qui s'établit entre le tuteur et l'apprenant. Divers émoticônes viennent renforcer cet aspect. Entre la première et la deuxième cohorte, on relève un usage plus répandu d'émoticônes variés, d'images fixes ou animées, en ouverture ou en clôture, mais aussi dans le corps du message, peut-être après en avoir vu l'usage dans certaines productions de la première cohorte. 
Figure 2. - Exemples d'avatars à visée socioaffective extraits des consignes de Carine.

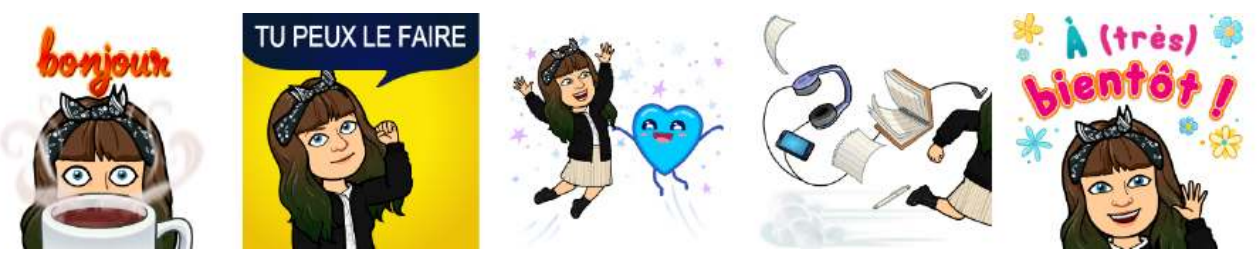

44 Ainsi, l'avatar de Carine se décline largement en salutations, encouragements ou encore émotions diverses, tandis qu'Agnès et Céline illustrent et renforcent leur message d'iconotexte, de gif animé (le petit chat - figure récurrente des technodiscours - qui salue) ou de photographie artistique, invitant à un état de sérénité.

Figure 3. - Exemples d'images fixes ou animées à visée socioaffective extraits des messages d'Agnès et de Céline.
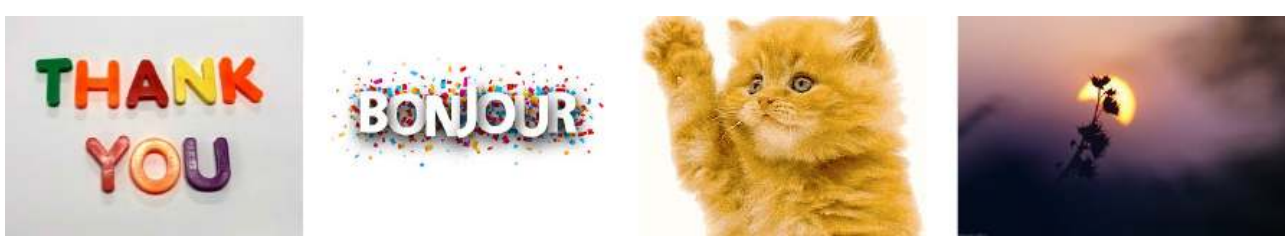

Par ailleurs, les messages de consigne sont organisés en paragraphes et la structure est rigoureuse. Ils servent à baliser ce que l'étudiant doit fournir comme travail au préalable. Les documents à étudier sont généralement joints dans le corps du message ou, pour une consultation plus optimale, en pièces jointes au format PDF ou Word (cet usage se retrouve particulièrement dans la deuxième cohorte). Un hyperlien est intégré pour que l'apprenant n'ait plus qu'à cliquer pour rejoindre la session de visioconférence. Le gras, mais aussi et surtout la couleur, sont employés pour mettre en évidence les parties importantes de la consigne dans les instructions ou alors viennent renforcer le contenu du message (par exemple bleu blanc rouge pour le mot « français », vert pour la thématique du développement durable).

Dans le schéma ci-dessous, nous mettons en exergue (dans les commentaires à gauche) la mise en discours numérique de la consigne et les différentes parties qui la structurent. 
Figure 4. - Capture d'écran d'un message de consigne d'Emmanuelle.

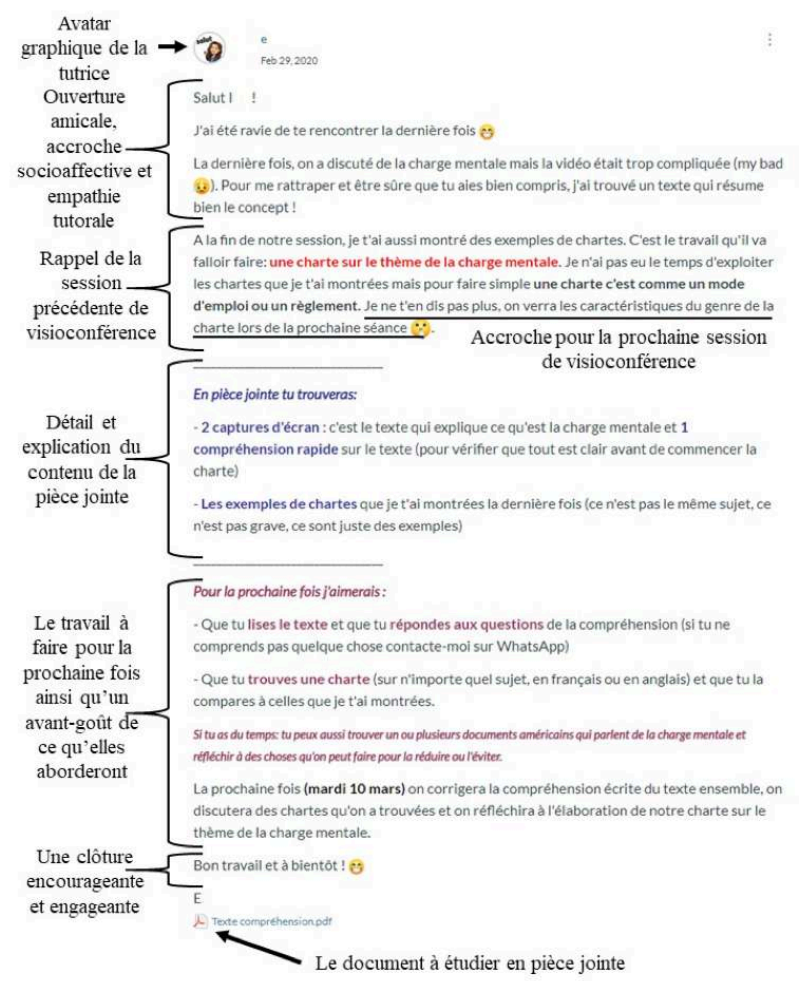

47 Ainsi, Emmanuelle montre qu'elle maitrise le genre de la consigne en ligne à partir des différents moments qui la caractérisent : des moments socioaffectifs, des moments cognitifs et injonctifs, plus ou moins renforcés sémiotiquement.

Enfin, outre le réinvestissement et la réappropriation, les étudiants participent à partir de genres sociaux existants à l'élaboration d'un genre tutoral nouveau : le selfie vidéo.

\subsection{Vers le remix et la création d'un genre tutoral original : le selfie vidéo}

Un selfie vidéo tutoral est un message de présentation de soi en tant que tuteur et une première prise de contact entre le tuteur et son apprenant en ligne. Il est, sur le plan théorique, intimement lié à l'étude du genre du vlogue et de la notion d'ethos. En tant que première prise de contact, il se doit d'être soigné même si marqué de la touche personnelle de l'étudiant. La télécollaboration débutant au semestre 2, il est préparé au semestre 1 et envoyé aux apprenants de FLE avant le début de leur cours. L'observation des différents selfies vidéos des tuteurs montre que les tuteurs se réapproprient des caractéristiques génériques du selfie et du vlogue, tout en inventant un genre tutoral original.

Tout d'abord, le choix du lieu de tournage est important: des lieux symboliques ou emblématiques pour l'étudiant (la nature, la ville d'étude, la chambre de l'étudiant). 
Figure 5. - Captures d'écran du selfie vidéo de Tabatha : lieux symboliques de la ville d'Aix-enProvence pour Tabatha.

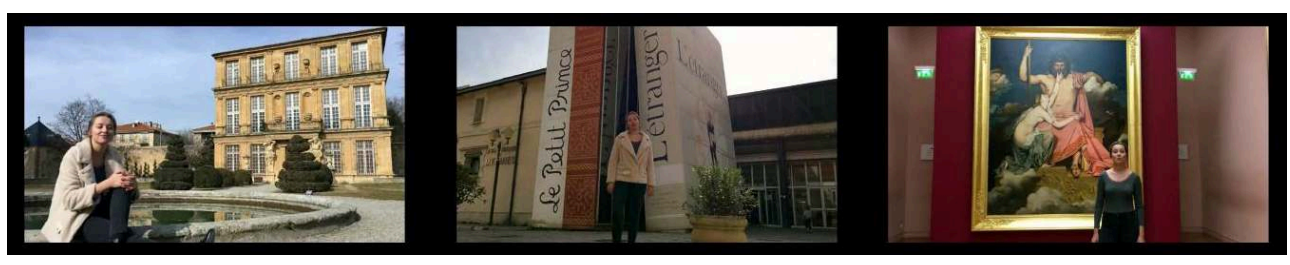

51 Ainsi, Tabatha a choisi de se filmer dans les différents lieux d'Aix qu'elle affectionne, mais également en lien avec ses goûts comme la lecture ou la peinture en posant dans les jardins d'un lieu culturel, devant la bibliothèque municipale ou dans un musée de la ville.

Emmanuelle répond à la voix off de la caméra qui l'interroge sur ses goûts « Langues? ... Les langues que je parle?»), tandis qu'Emma s'adresse à son apprenant, dans une forme de vlogue intimiste, comme le soulignent le choix du lieu de tournage (sa chambre) et le cadrage (en gros plan).

Figure 6. - Captures d'écran du selfie vidéo d'Emmanuelle et d'Emma.

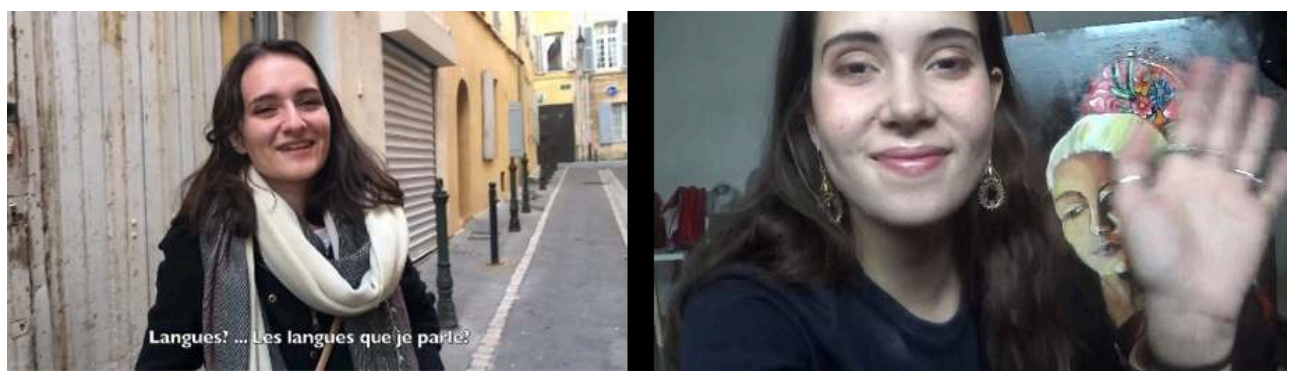

53 Trois étudiantes de la première cohorte ont fait un montage humoristique mettant en évidence les ratés de leur tournage ${ }^{11}$. Dans certaines vidéos de la deuxième cohorte, on observe davantage l'usage de sous-titres en français ou plurilingues. En effet, dans un contexte d'enseignement à un public allophone, un sous-titrage des paroles peut être bienvenu pour un premier contact et aider la compréhension ou encore faciliter la pratique sociale commune de la lecture silencieuse de vidéo en ligne.

Figure 7. - Captures d'écran des selfies vidéo d'Agnès et Céline.

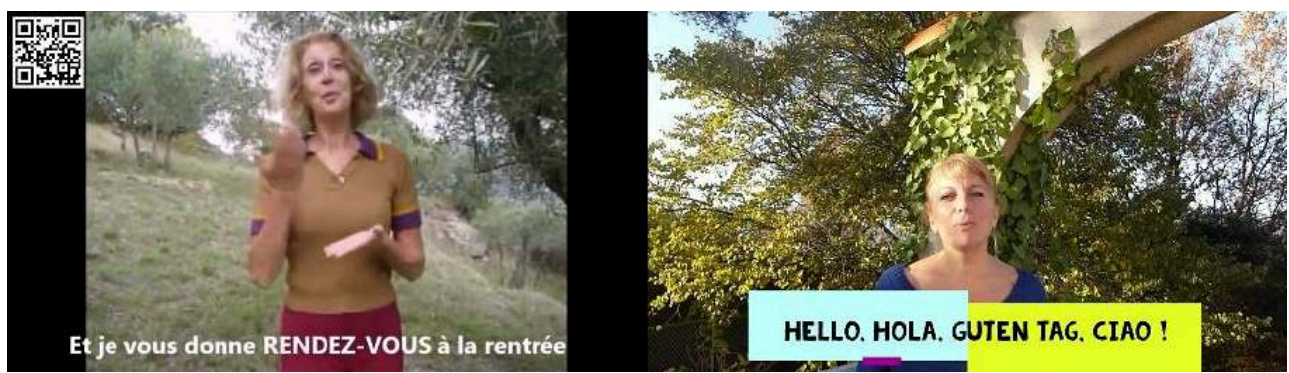

Deux étudiantes ont élaboré un scénario plus complexe. La première, Tabatha, a construit son selfie vidéo comme un reportage à travers des lieux de la ville qu'elle affectionne (cf. fig. 5). La seconde, Carine, en s'inspirant d'une de ses journées, a produit un court métrage très dynamique et aux nombreuses incrustations graphiques, comme on peut le voir dans les captures d'écran ci-dessous. 
Figure 8. - Captures d'écran du selfie vidéo de Carine : déroulé de sa journée et lieux symboliques de la vie étudiante.

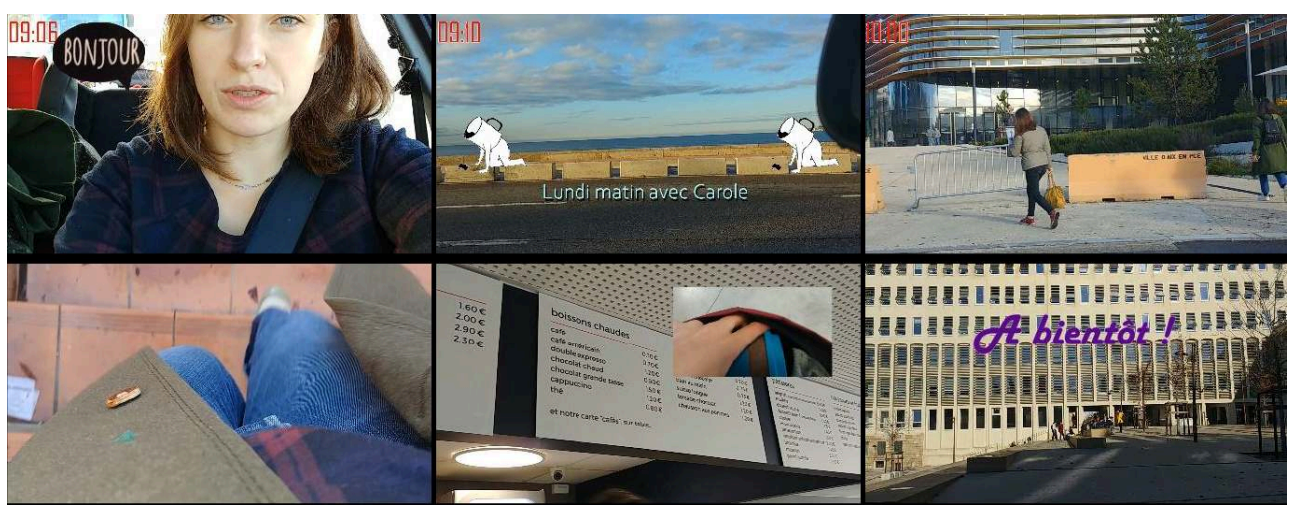

\section{Discussion}

55 À la lumière des analyses précédentes, il apparait que les étudiants en didactique qui sont formés à l'ADN s'approprient les concepts et qu'à l'aide de ces concepts, ils analysent mieux d'un point de vue descriptif-compréhensif mais aussi critique les documents numériqués auxquels ils sont confrontés. Par ailleurs, s'appuyant sur les productions des cohortes antérieures, mais aussi sur leurs pratiques sociales, ils exercent leur créativité et contribuent à formaliser les genres numériques de la fonction enseignante qui sont en train d'émerger, tels le selfie vidéo tutoral, la consigne en ligne sur un forum de discussions ou encore le e-portfolio de développement professionnel. Même si les genres numériques sont encore en cours de définition, les étudiants peuvent s'appuyer sur leurs pratiques sociales et transférer dans leur pratique enseignante les connaissances qu'ils ont de certains genres comme le selfie ou le vlog pour le selfie vidéo tutoral par exemple.

Enfin, nous tenons aussi à souligner l'importance du réinvestissement possible du développement de ces compétences technosémiodiscursives et de cette formation par genre numérique dans la création de scénarios pédagogiques originaux intégrant le numérique. Ainsi, nous souhaitons inscrire cette réflexion dans la continuité des travaux de Claudel et Laurens (2016) qui préconisent le recours à la notion de genre discursif à l'aide de l'analyse du discours dans la formation à la didactique des langues. Ces auteurs proposent, à partir de la connaissance d'un genre donné, d'aborder la didactisation des documents authentiques par différentes entrées (linguistique, pragmatique, culturel, etc.), mais omettent l'aspect numérique des discours ou des genres (y compris pour l'exemple choisi du message électronique). Or, il nous semble que c'est principalement sur Internet que les futurs enseignants iront puiser leurs documents authentiques: une approche par genres numériques et une prise en considération des aspects de littératie numérique, y compris dans des scénarios pédagogiques en didactique des langues, peut donc s'avérer intéressante, voire indispensable. En effet, des compétences en littératie numérique peuvent être non seulement une entrée pour la sélection de documents authentiques mais aussi une compétence transversale à développer au sein du scénario pédagogique au même titre que par exemple le développement de l'autonomie, du savoir-apprendre ou du savoirêtre des apprenants. Les questions de la vie privée et de la sécurité en ligne, de la 
gestion de l'identité numérique, de la cyberviolence, de l'empreinte numérique, de la publicité et de la consommation en ligne, de l'information et de la vérification des sources sur Internet, de la création et du remixage de contenu numérique sont autant d'aspects possibles à traiter.

Ainsi, dans un scénario pédagogique à partir du genre du e-portfolio sur LinkedIn, les compétences suivantes pourront être visées :

Tableau 2. - Exemple de compétences à travailler à partir du genre du e-portfolio.

\begin{tabular}{|c|c|c|c|c|}
\hline Pragmatique & Linguistique & Sociolinguistique & $\begin{array}{l}\text { Vie privée et } \\
\text { sécurité }\end{array}$ & Savoir-faire \\
\hline $\begin{array}{l}\text { Décrire son } \\
\text { parcours, ses } \\
\text { expériences. }\end{array}$ & $\begin{array}{l}\text { Maitriser } \\
\text { l'usage et la } \\
\text { conjugaison du } \\
\text { passé } \\
\text { composé. }\end{array}$ & $\mid \begin{array}{lrr}\text { Prendre contact } & \text { de } \\
\text { manière simple } & \text { et } \\
\text { efficace avec de futurs } & \\
\text { employeurs } & \\
\text { en respectant } & \text { les } \\
\text { usages de base. } & \end{array}$ & 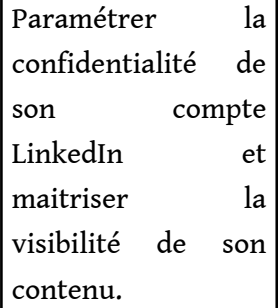 & \begin{tabular}{|lr} 
Trouver & des \\
collaborateurs, & \\
s'affilier à & un \\
réseau & (d'anciens \\
élèves & par \\
exemple). &
\end{tabular} \\
\hline
\end{tabular}

Outre que toutes s'inscrivent dans un espace de production discursive numérique (en l'occurrence celui de LinkedIn), la quatrième relève plus spécifiquement de la littératie numérique.

\section{Conclusion}

Dans cet article, nous souhaitions montrer l'intérêt de recourir au nouveau cadre de l'ADN et de la notion de genre numérique et comment ce cadre peut servir la formation à la littératie numérique de futurs enseignants de FLE et s'inscrire dans la lignée de recherches antérieures en didactique du FLE et analyse du discours. On a pu observer que les étudiants, à partir des concepts de l'ADN, étaient à même d'analyser en détail des documents numériqués, de les inscrire dans un genre numérique puis d'en produire eux-mêmes à leur tour. Ainsi, ces futurs professionnels du FLE ont développé des compétences technosémiolinguistiques qu'ils pourront transférer en compétences technosémiopédagogiques dans le choix et l'analyse, d'une part, des documents numériqués qu'ils exploiteront avec leurs apprenants, mais aussi dans la maitrise, d'autre part, des différents genres numériques tutoraux qu'ils seront amenés à expérimenter. Enfin, soulignons l'intérêt d'élaborer dans de futurs travaux une cartographie descriptive des genres numériques de la vie sociale, mais aussi des genres numériques propres à la fonction enseignante. 


\section{BIBLIOGRAPHIE}

BARDIN, Laurence. (2013). L'analyse de contenu. Presses universitaires de France. <https://doi.org/ 10.3917/puf.bard.2013.01>.

CAPPELLINI, Marco \& ComBE, Christelle. (2017). Analyser des compétences techno-sémiopédagogiques d'apprentis tuteurs dans différents environnements numériques : résultats d'une étude exploratoire. Alsic, 20(3). <https://doi.org/10.4000/alsic.3186>.

CLAUDEL, Chantal. (2017). Enseignement des langues étrangères : épistémologie, théorisation et discours ordinaires. Les Carnets du Cediscor, 13, 19-32. <https://doi.org/10.4000/cediscor.1006>.

Claudel, Chantal \& LAURENS, Véronique. (2016). Le genre discursif comme objet d'enseignement en didactique du français. SHS Web of Conferences (Actes du $5^{e}$ Congrès mondial de linguistique française), 27. <www.shs-conferences.org/articles/shsconf/pdf/2016/05/ shsconf_cmlf2016_07005.pdf>.

COMBE CELIK, Christelle. (2010). Pratiques discursives dans une formation en ligne à la didactique du français en langue étrangère (Thèse de doctorat). Université StendhalGrenoble 3, Grenoble. <https://tel.archives-ouvertes.fr/tel-00508363/>.

СомBE, Christelle. (2019). Les genres numériques de la relation. Langage et société, 167, 51-80. <https://doi.org/10.3917/ls.167.0051>.

COMBE, Christelle. (2020). Former de futurs professionnels du FLE au genre du e-portfolio : une étude exploratoire. Le numérique dans l'enseignement supérieur. Dans N. Rentel \& P. von Münchow (dir.), Enjeux et défis du numérique pour l'enseignement universitaire / Chancen und Herausforderungen der Digitalisierung in der Hochschullehre (p. 93-117). Peter Lang.

COMBE, Christelle \& CAPPELLINI, Marco. (2021). La construction d'un ethos discursif multimodal de futurs enseignants en ligne. Le français dans le monde. Recherches et applications, 69, 33-45.

CRYSTAL, David. (2011). Internet Linguistics: A Student Guide. Routledge.

DeVElotTe, Christine. (2006). Décrire l'espace d'exposition discursive dans un campus numérique. Le français dans le monde. Recherches et applications, 40, 88-100. <http://halshs.archives-ouvertes.fr/ docs/00/15/18/51/PDF/expositiondiscursive.pdf>.

DeVelotTe, Christine \& MANGENOT, François. (2010). Former aux tutorats synchrone et asynchrone en langues. Distances et savoirs, 8(3), 345-359.

Frau-MeIGS, Divina, O’NEILl, Brian, Soriani, Alessandro \& TomÉ, Vitor. (2017). Digital Citizenship Education. Overview and New Perspectives. Council of Europe Publishing. <https://rm.coe.int/ prems-187117-gbr-2511-digital-citizenship-literature-review-8432-web1/168077bc6a>.

GuICHON, Nicolas. (2012). Vers l'intégration des TIC dans l'enseignement des langues. Didier.

JeWITT, Carey, BEZEMER, Jeff \& O’HALloRAN, Kay. (2016). Introducing Multimodality. Routledge.

KARSENTI, Thierry \& ColLIN, Simon. (2012). Le portfolio électronique en formation initiale des enseignants. Actes de la Recherche, 9, 81-94.

LACELLE, Nathalie, Boutin, Jean-François \& LEBrun, Monique. (2018). La littératie médiatique multimodale appliquée en contexte numérique. Presses de l'Université du Québec. 
LEBRUN, Monique, LACELLE, Nathalie \& Boutin, Jean-François. (2012). La littératie médiatique multimodale: de nouvelles approches en lecture-écriture à l'école et hors de l'école. Presses de l'Université du Québec.

MAINGUENEAU, Dominique. (2012). Que cherchent les analystes du discours ? Argumentation et analyse du discours, 9. <https://doi.org/10.4000/aad.1354>.

MANGENOT, François. (2017). Formation en ligne et MOOC : apprendre et se former en langue avec le numérique. Hachette FLE.

OLLIVIER, Christian. (2018). Littératie numérique et approche socio-interactionnelle pour l'enseignementapprentissage des langues. Éditions du Conseil de l'Europe. <www.ecml.at/Portals/[...]/ECMLresources/elang-FR-A5_28112018_112746.pdf>.

PaVeau, Marie-Anne. (2015). Ce qui s'écrit dans les univers numériques. Matières technolangagières et formes technodiscursives. Itinéraires. <https://doi.org/10.4000/itineraires. 2313>.

PAVEAU, Marie-Anne. (2017). L'analyse du discours numérique. Dictionnaire des formes et des pratiques. Hermann.

THE NeW London GRoup. (1996). A Pedagogy of Multiliteracies: Designing Social Futures. Harvard educational review, 66(1), 60-93.

\section{NOTES}

1. Nous considérons ce passage comme un prolongement naturel à l'ère de la globalisation et de la numérisation généralisée de nos sociétés alors que les recherches en FLE tendent désormais à s'inscrire dans le champ de la DDL avec pour principe la reconnaissance de la diversité linguistique et culturelle et qu'Internet est, par ailleurs, multilingue (Crystal, 2011) et un espace propice au développement du plurilinguisme.

2. Publié pour la première fois en 2017, en anglais, sous le titre European Framework for the Digital Competence of Educators: DigCompEdu, par le Joint Research Centre de la Commission européenne. Cf. <http://europa.eu/!gt63ch>.

3. «L'extimité consiste sur internet, et tout particulièrement sur les réseaux sociaux numériques du web participatif, en l'extériorisation de l'intimité des internautes à des fins de validation de l'image de soi. » (Paveau, 2017, p. 185)

4. Nous désignons par l'expression « document numériqué » (Paveau, 2015) tout document natif du numérique.

5. Nous définissons le scénario pédagogique comme la conceptualisation didactique par l'enseignant de la future mise en œuvre pédagogique. Il prend en compte le contexte (public cible et niveau langagier), présente les compétences visées (langagières, plurilingues et pluriculturelles, de littératie numérique, de savoir-être, de savoir-apprendre, etc.) pour réaliser la tâche finale, ainsi que les différentes étapes de cette réalisation pour y parvenir. Il spécifie le design pédagogique, les outils numériques et les modalités d'évaluation.

6. La démarche portfolio est approfondie et le e-portfolio complété dans le cours «Évaluer et certifier en DDL » au second semestre puis tout au long de l'année de master 2 dans le cours " Développer un agir professoral réflexif ».

7. C'est aussi l'occasion de leur rappeler l'importance de partir de l'activité numérique sociale de leurs futurs apprenants pour créer leur scénario pédagogique.

8. Nous surlignons en gras.

9. Nous surlignons en gras. 
10. Moodle est la plateforme d'enseignement en ligne de l'université.

11. Pour une analyse détaillée de la première cohorte, se reporter à Combe et Cappellini (2021).

\section{RÉSUMÉS}

Cet article s'appuie sur les notions de littératie médiatique, multimodale et numérique et s'ancre dans le cadre conceptuel de l'analyse du discours numérique et notamment de la notion centrale de genre numérique. Il montre comment des étudiants de master de FLE s'emparent des différents éléments de la formation à la littératie numérique à travers notamment l'analyse du discours numérique et l'appropriation de trois genres numériques émergents de la fonction enseignante : le selfie vidéo tutoral, la consigne en ligne et le e-portfolio de développement professionnel.

This article is based on the notions of media, multimodal and digital literacy and is rooted in the conceptual framework of digital discourse analysis and in particular the central notion of digital genre. Through digital discourse analysis and the appropriation of three emerging digital genres related to teaching-the tutor selfie video, online instruction-giving and the professional development e-portfolio-this article shows how FFL master's students take hold of the different elements of digital literacy training.

\section{INDEX}

Mots-clés : littératie numérique, genres numériques, ethos tutoral, consigne en ligne, selfie vidéo tutoral, e-portfolio de développement professionnel

Keywords : digital literacy, digital genres, tutor ethos, online instruction-giving, tutor selfie video, professional development e-portfolio

\section{AUTEUR}

\section{CHRISTELLE COMBE}

Aix Marseille Université, CNRS, LPL, Aix-en-Provence, France

christelle.combe@univ-amu.fr 